\title{
REFLECTIONS
}

\section{Cherry Blossoms, COVID-19, and the Opportunity for a Healthy Life}

\author{
Marsball H. Chin, MD, MPH \\ Section of General Internal Medicine, \\ Department of Medicine, University \\ of Chicago, Chicago, Illinois
}

\begin{abstract}
To date, short-term funding and policy fixes for the coronavirus disease 2019 (COVID-19) pandemic have focused on saving the current health care system; policies have not maximized the population's health, prioritized the safety net, nor addressed the fundamental problems that have hindered our nation's response for our most vulnerable neighbors. We need to plan more lasting equity-specific reforms now. I explain 3 lessons that should inform reforms to the health care delivery and payment systems to reduce health disparities and maximize the public's health: (1) Proven roadmaps and processes for reducing health care disparities already exist, as do themes of successful interventions. Implement them; (2) Payment reform needs to create a business case for health care organizations to address social determinants of health and implement care interventions to reduce health disparities; (3) We as a nation need to have hard conversations about whether we truly value the opportunity for everyone to have a healthy life.
\end{abstract}

Ann Fam Med 2021;19:63-65. https://doi.org/10.1370/afm.2630.

S earching for peace and beauty in our coronavirus disease 2019 (COVID-19) world, my wife and I walked daily to the Japanese cherry blossoms around the placid pool of water near the Museum of Science and Industry on the South Side of Chicago. Over a 3-week period, pink cherry blossoms grew ever more radiant, becoming our highlight each day. But the cherry blossoms eventually faded and dropped to the ground. Cherry blossoms have been a metaphor for life in haiku poetry, beautiful and ephemeral, ultimately withering into death.

Japanese cherry blossoms are also a metaphor for our nation's response to the disproportionate harm COVID-19 is inflicting on low-income and racial and ethnic minority communities. To meet the immediate medical and social needs exposed by the COVID-19 epidemic, state and federal governments have rapidly responded with stopgap funding and incremental policy fixes. While flawed, this funding has still been helpful and attracted many applicants like bees to nectar ${ }_{i}$ however, this funding will gradually fade away like cherry blossoms. Now is the time to address the fundamental structural problems in the health care delivery and payment systems that perpetuate health disparities.

\section{MORE OF THE SAME OR TRUE REFORM}

In the United States, public and health care organizations have been overwhelmed by the medical, social, and financial stresses of dealing with COVID-19. Two fundamental questions implicitly hover over the immediate health policy relief efforts: (1) Should the goal of aid be to help the patients most adversely impacted by COVID-19 or to should the aid be used to save the current health care system? (2) Should the nation prop up the status quo or encourage a health care system better designed to
Marshall H. Chin

University of Chicago

Section of General Internal Medicine 5841 South Maryland Avenue, MC2007 Chicago, Illinois 60637 mchin@medicine.bsd.uchicago.edu 
address the complex medical and social needs of highrisk populations? The de facto answer has been to save the current health care system, not to maximize the population's health, not to prioritize the safety net, and not to address the fundamental problems that have hindered our nation's response to COVID-19 for our most vulnerable neighbors.

If helping the patient was the primary goal, we would have improved access to health care and COVID-19 testing by expanding Medicaid and by reopening all the Patient Protection and Affordable Care Act health insurance exchanges to allow the newly unemployed to enroll in a health plan. Instead, $\$ 50$ billion of the initial CARES Act Provider Relief Fund were allocated to support the current health care system by giving hospitals and providers funding "to support health care-related expenses or lost revenue attributable to COVID-19 and to ensure uninsured Americans can get testing and treatment for COVID-19."1 The funding formulas favored big hospitals rather than safety-net providers, based on market shares of Medicare costs and total patient revenue. ${ }^{2}$ Key regulatory changes to Medicare to improve access to care, such as increased and expanded reimbursement of telehealth by physicians, nurse practitioners, mental health professionals, and physical therapists are temporary.

Incremental, short-term, stopgap measures reflect urgent logistical and political realities, such as helping patients in critical need and keeping small, rural practices open. It would be a mistake if policy makers, key stakeholders, and the public stop there. They should consider what health care system and payment reforms would care for vulnerable populations more effectively both in times of extreme stress such as the COVID-19 pandemic and routinely. It is short-sighted and unconscionable to not address the root drivers of inequities and the structural problems in the health and social service systems that have contributed to the disparate suffering, burden, and death in low-income and racial and ethnic minority populations.

\section{LESSONS TO REDUCE HEALTH DISPARITIES}

What lessons do we already know that can inform reforms to the health care delivery and payment systems that will reduce health disparities and maximize the public's health?

\section{Implement Proven Roadmaps and Processes and Successful Interventions For Reducing Health Care Disparities}

It is not a magical or impossible task to reduce health disparities. ${ }^{3,4}$ Expand health insurance coverage.
Identify disparities in health care quality and outcomes data. Do a root cause analysis that includes speaking with the affected populations to understand why the disparities exist, then design and implement interventions that address the causes. Revise and improve over time. Culturally tailored team-based care involving close monitoring of patients, multiple human touches, and social connections reduces disparities. Bridge the worlds of the health care system and community, involving patients, community health workers, and communities as partners, and address social factors that impact health outcomes. In addition, increasing the racial and ethnic diversity of the health care workforce can improve health disparities by increasing access to care and quality of care for racial and ethnic minority populations. ${ }^{5,6}$

\section{Create a Business Case for Health Care Organizations to Address Social Determinants of Health and Implement Care Interventions to Reduce Health Disparities}

Even the most well-meaning organizations have difficulty sustaining interventions to reduce disparities without a business case. ${ }^{7}$ In the Robert Wood Johnson Foundation Advancing Health Equity: Leading Care, Payment, and Systems Transformation program, we are working with teams consisting of a state Medicaid agency, a Medicaid managed-care organization, and frontline health care delivery organizations to enact payment reform that supports and incentivizes the care delivery changes that reduce health disparities. Stakeholders must align their efforts. Each team is considering how to provide flexible upfront funding (eg, capitation, per-member per-month payments) for care interventions that reduce disparities such as community health workers and culturally tailored team-based care. Each team is also considering how value-based payment programs can use performance metrics to incentivize and reward reduction of disparities (eg, pay for reducing disparities; measures sensitive to addressing social determinants of health such as preventable hospitalizations for ambulatory care-sensitive conditions). In the Merck Foundation Bridging the Gap: Reducing Disparities in Diabetes Care program, Marshall University has worked with Appalachian federally qualified health centers and payers to fund community health workers with a shared savings model. ${ }^{8}$ Regardless of whether fee-for-service, value-based payment, or alternative payment models are used, the approaches must be intentionally designed to support and incentivize the reduction of health disparities. Currently there are few or weak incentives in most value-based payment schemes and alternative payment models to reduce disparities. ${ }^{7}$ 


\section{Have the Hard Conversations About Whether We Truly Value the Opportunity for Everyone to Have a Healthy Life}

Care delivery and payment reforms are necessary to reduce health disparities, but will not happen unless we have a culture of equity in which we truly value giving everyone a fair chance for a healthy life. We need to have free, frank, and fearless discussions about the structural racism and systems of privilege that have caused racial and ethnic minorities and low-income communities to suffer health disparities, have worse housing, worse education, and less economic opportunity, and commit to leveling the playing field. ${ }^{9}$ Diverse crowds are repeatedly protesting police brutality against Black Americans, reflecting an increased willingness of our society to discuss systemic racism and advocate for change. Specific suggestions for increasing the effectiveness of conversations around racism and inequities include leading with stories not numbers, not blaming the individuals in the discussions, teaching about systemic solutions rather than only problems, and discussing concrete ways each person can advocate for reform. ${ }^{10,11}$

\section{A CALL TO ACTION LOCALLY AND NATIONALLY}

For example, we can look in the mirror and implement anchor institution principles at our home organizations, such as hiring neighborhood residents and using community vendors to improve the local economy, and screening routinely for social needs in our patients. ${ }^{12}$ We can insist that our clinics and hospitals improve their quality of care with an equity lens, designing their systems to specifically meet the needs of diverse patients including the most vulnerable. ${ }^{4}$ And we can mobilize support in our professional societies to advocate for payment reforms that intentionally support and reward health care organizations that address social determinants of health and advance health equity. ${ }^{7}$

The Cities of Osaka and Chicago partnered to revitalize an exquisitely beautiful, peaceful Japanese Garden of the Phoenix near the Museum of Science and Industry to symbolize friendship and respect. On our walks, my wife and I pass by a stone tōrō lantern near the fading blossoms. The stone structure has strength and beauty that are permanent and timeless. Our nation has the potential to unite and commit to long lasting care delivery and payment reforms to advance health equity. Similar to the Greek legend of the phoenix, good can arise out of the destruction from the COVID-19 pandemic. In Chinese and
Japanese cultures, the phoenix appears in virtuous times; it is up to us to ensure that everyone can have a fair and just opportunity to live a healthy life.

To read or post commentaries in response to this article, see it online at https://www.AnnFamMed.org/content/19/1/63.

Submitted May 19, 2020; submitted, revised July 1, 2020; accepted July 21, 2020.

Funding support: Dr Chin was supported in part by the Robert Wood Johnson Foundation Advancing Health Equity: Leading Care, Payment, and Systems Transformation Program Office, the Merck Foundation Bridging the Gap: Reducing Disparities in Diabetes Care National Program Office, and the Chicago Center for Diabetes Translation Research (NIDDK P30 DK092949).

Key words: equity; disparities; payment; quality of care; COVID-19

\section{References}

1. Department of Health and Human Services. CARES Act Provider Relief Fund. Accessed May 7, 2020. https://www.hhs.gov/ coronavirus/cares-act-provider-relief-fund/index.html

2. Feinberg $A$. The $\$ 175$ million fund meant for health care providers treating coronavirus could be a windfall for big hospital chains instead. Newsweek. Published Apr 27, 2020. Accessed Jul 1, 2020. https://www.newsweek.com/175-billion-fund-meant-health-careproviders-treating-coronavirus-could-windfall-big-hospital-1500171

3. National Academies of Science, Engineering, and Medicine. Systems Practices for the Care of Socially At-Risk Populations. Washington, DC: The National Academies Press;2016. 10.17226/21914.

4. Chin MH, Clarke AR, Nocon RS, et al. A roadmap and best practices for organizations to reduce racial and ethnic disparities in health care. J Gen Intern Med. 2012;27(8):992-1000.

5. Marrast LM, Zallman L, Woolhandler S, Bor DH, McCormick D. Minority physicians' role in the care of underserved patients: diversifying the physician workforce may be key in addressing health disparities. JAMA Intern Med. 2014;174(2):289-291.

6. Cooper LA, Roter DL, Johnson RL, Ford DE, Steinwachs DM, Powe NR. Patient-centered communication, ratings of care, and concordance of patient and physician race. Ann Intern Med. 2003;139(11): 907-915.

7. DeMeester RH, Xu LJ, Nocon RS, Cook SC, Ducas AM, Chin MH. Solving disparities through payment and delivery system reform: a program to achieve health equity. Health Aff (Millwood). 2017; 36(6):1133-1139.

8. Crespo R, Christiansen M, Tieman K, Wittberg R. An emerging model for community health worker-based chronic care management for patients with high health care costs in rural Appalachia. Prev Chronic Dis. 2020;17:E13. https://doi.org/10.5888/pcd17.190316

9. Chin $\mathrm{MH}$, King $\mathrm{PT}$, Jones RG, et al. Lessons for achieving health equity comparing Aotearoa/New Zealand and the United States. Health Policy. 2018;122:837-853. pii: S0168-8510(18)30131-3. 10.1016/j.healthpol.2018.05.001

10. Peek ME, Vela MB, Chin MH. Practical lessons for teaching about race and racism: successfully leading free, frank, and fearless discussions. Acad Med. 2020;95(12S):S139-S144.

11. Tan JY, Baig AA, Chin MH. High stakes for the health of sexual and gender minority patients of color. J Gen Intern Med. 2017;32(12): 1390-1395. 10.1007/s11606-017-4138-3

12. Chen $\mathrm{AH}$, Chin $\mathrm{MH}$. What if the role of healthcare was to maximize health? J Gen Intern Med. 2020;35(6):1884-1886. 10.1007/ s11606-019-05524-3 